\title{
Additive Manufacturing of Ceramics
}

\author{
Teddy Slowik ${ }^{l}$ \\ ${ }^{1}$ Woodbury University \\ ${ }^{I}$ teddy@novatona.com
}

\begin{abstract}
Additive manufacturing (AM) is vastly developing across the industrial landscape and has recently expanded outside of the traditional polymeric and metallic-based materials. Ceramics are an ever-present material in the architectural field, but there has been minimal evolution in its associated manufacturing processes. The limitations of additive manufacturing of ceramics are quickly evolving and will soon create new potentials for architectural products and applications. This paper offers an overview of these limitations that are tested and examined through a case study.
\end{abstract}

Keywords: Additive Manufacturing, Ceramics, Robocasting, 3D Printing

\section{INTRODUCTION}

Ceramics are a novel material that have been utilized in constructed environments for centuries, and until recently have been restricted to traditional modes of craft when applied at the scale of architecture. Casting and extruding have been the primary methods of ceramics manufacturing in the building materials industry. These traditional manufacturing techniques have limited the potential of ceramics across the allied design fields. In observation of rapid advancements in digital systems of production and fabrication, it only feels appropriate to apply these advancements to a material that has been ever-present yet primitive in the field of architecture. Hybridizing conventional methods of production with the rapidly expanding arsenal of digital tools available today will open new avenues for ceramic applications in the field of architecture.

Ceramics are an inherently attractive building material due to the rare combination of exceptional mechanical, thermal and chemical properties (Faes 2015). However, traditional modes of manufacturing in the ceramics industry have limited the poten- tial for mass-customized components. High costs associated with unique molds and lengthy production timeframes are just a few of the constraints that have stifled the production of geometrically complex ceramic components. "The production of highly complex 3D shapes, micro features, or structures with tailored porosity, such as scaffolds, is still seen as a major limit" (Faes 2015). Additive manufacturing of engineered ceramics can vastly expand the current range of production possibilities.

\section{ADDITIVE MANUFACTURING PROCESSES}

Up until this point, most work and research on additive manufacturing have been centered on polymers and metals. Additive manufacturing of ceramics is significantly less developed, and therefore ripe with potential for further investigation and research. There are several additive manufacturing processes that are currently being utilized and developed in the ceramics industry. Stereolithography (SLA), Lithography-based Ceramic Manufacturing (LCM), Freeze-Form Extrusion (FFE), Selective Laser Sintering (SLS), Fused Deposition of Ceramics 

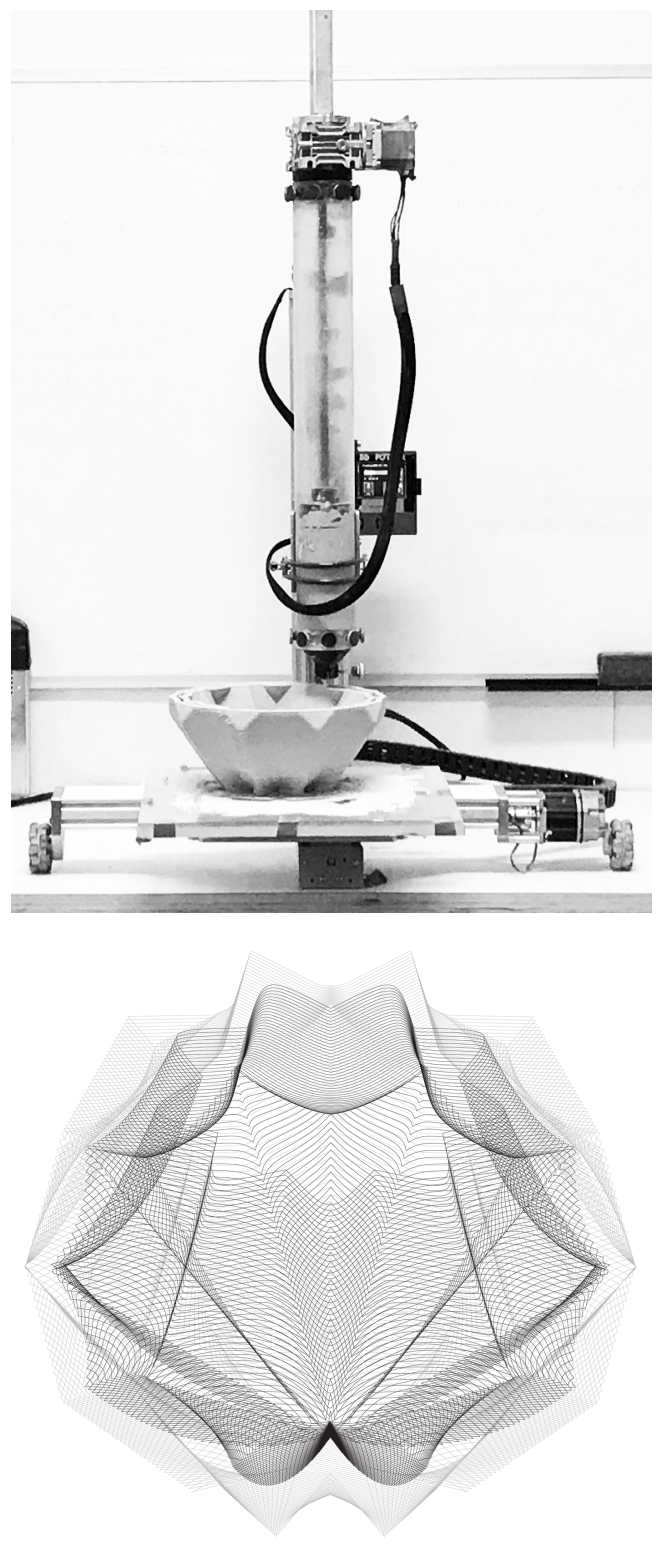

(FDC) and Robocasting have all been investigated for their feasibility in the production of ceramic components (Faes 2015). Robocasting will be the focal process in the case studies outlined in this paper.

Robocasting is an additive manufacturing technique in which a filament or paste is extruded from a nozzle onto a fixed or dynamically controlled platform. Objects are produced by adding material in a layer-by-layer fashion where either the nozzle is moved up or the platform is moved down. Unlike many consumer grade 3D printers that print via fused deposition of thermoplastic filaments, robocasting relies on surface tension to fuse each layer together (Feilden 2016). There are no thermal gradients involved during the robocasting process, and the extrusion pressures are much less than fused deposition modeling (Feilden 2016).

\section{CALIBRATION CONSTRAINTS}

There are many variables to consider in the robocasting manufacturing process of ceramics. The stepover and angle of cantilever without additional formwork or support structure is an ever-present constraint. In fused deposition thermoplastic prints, 45 degrees tends to be the maximum angle one can achieve without secondary support structure. These constraints were investigated over a series of case studies conducted with a 3D PotterBot from DeltaBots. A concentric multifaceted geometry was designed in Rhinoceros to specifically test and calibrate an array of material constraints. The subject artifact's geometry is a composition of curved and flat surfaces that both undulate and cantilever at various degrees. Simplify3D was utilized as the slicing software to generate the g-code for the 3D PotterBot. Paste composition and environmental conditions during the printing and drying period had major effects on these tests. Several trial prints with various sized nozzles were conducted, and subsequently a $4 \mathrm{~mm}$ circular nozzle was found suitable due to its consistency. A z-step of $2 \mathrm{~mm}$ was found to provide sufficient adherence to the previous layer. With these calibrations, a maximum cantilever of 30 de-
Figure 1

3D PotterBot in the process of printing

Figure 2

G-code line drawing of a $2 \mathrm{~mm}$ z-step 
Figure 3

30 degree cantilever from base that rises $4 "$ grees was achieved over a four-inch tall outward sloping concentric print; significant slumping and failure occurred in cantilevers larger than 30 degrees. Further, secondary supports must be printed or introduced to support most cantilevers and overhangs. Depending on the paste composition, printing speed and printing environment, robocasting can typically deal with large spanning regions many times the filament diameter in length, where the structure is unsupported from below (Smay 2002).

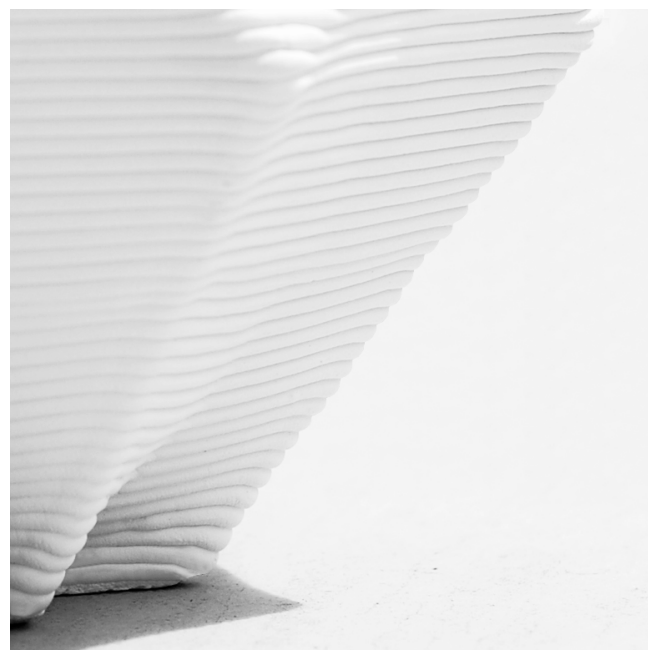

Additionally, paste composition is fundamental to a successful robocasted print. The slurry or paste must be suitably homogenous and free of air bubbles and agglomerates (Feilden 2017). To eliminate air bubbles from the ceramic paste and ensure a consistent paste a de-airing pugmill was utilized to process the paste for our investigations. This tool utilizes a vacuum to remove air bubbles from the paste and extrudes a consistent cylindrical volume of paste directly into a clear plastic tube which is then loaded into the 3D PotterBot. Even when processing the paste with the de-airing pugmill a low percentage of air bubbles remained and produced inconsistencies in each print. The tube's volume capacity proves to be a constraint because without a continuous feed significant drying of the clay occurs during the unloading and loading of each tube into the PotterBot's RAM extruder. This results in the new layer not bonding uniformly to the last layer of the previous tubes print. The z-height calibration when loading a new tube also proves to be troublesome when attempting to print with multiple tubes as the last clay layer settles during the tube interchange. This produces a noticeable inconsistency in the horizontal banding at this layer. This inconsistent banding is problematic both visually and in performance. Therefore, our studies were limited to single tube print volumes under $1500000 \mathrm{~mm} 3$.

Furthermore, the viscosity of the paste must be low enough to allow for extrusion through the printer's nozzle, but stiff enough to hold its shape and allow each new layer to bind with the previous layer. The ratio of ceramic powder to water must be high in order to achieve high green densities which reduces drying shrinkage and allows complete sintering (Feilden 2017). Pastes containing up to $60 \%$ volume of ceramic particles have been successfully employed (Faes 2015). There have been several studies executed on highly engineered pastes with additives such as gels, resins, deflocculants and fibers to ensure a high degree of printability with minimal shrinkage (Feilden 2016). Aqueous pastes are desirable due to their simplicity, lower cost, low toxicity and slower drying periods (Feilden 2016). Therefore, in our case studies we focused solely on aqueous pastes and developing an appropriate ceramic powder to water ratio.

\section{MATERIAL AFFECTS}

One of the unfortunate results of utilizing a $4 \mathrm{~mm} \mathrm{noz-}$ zle tip with a $2 \mathrm{~mm}$ z-step is the distinct staircasing affect that is produced via this layered manufacturing process. Additionally, there are a number of defects that are fairly commonplace in the manufacturing and processing of ceramics: agglomerates, bubbles, contaminates/inclusions, and large grains occur quite frequently (Feilden 2017). Subsequent machining in the green unfired stage is required to achieve 

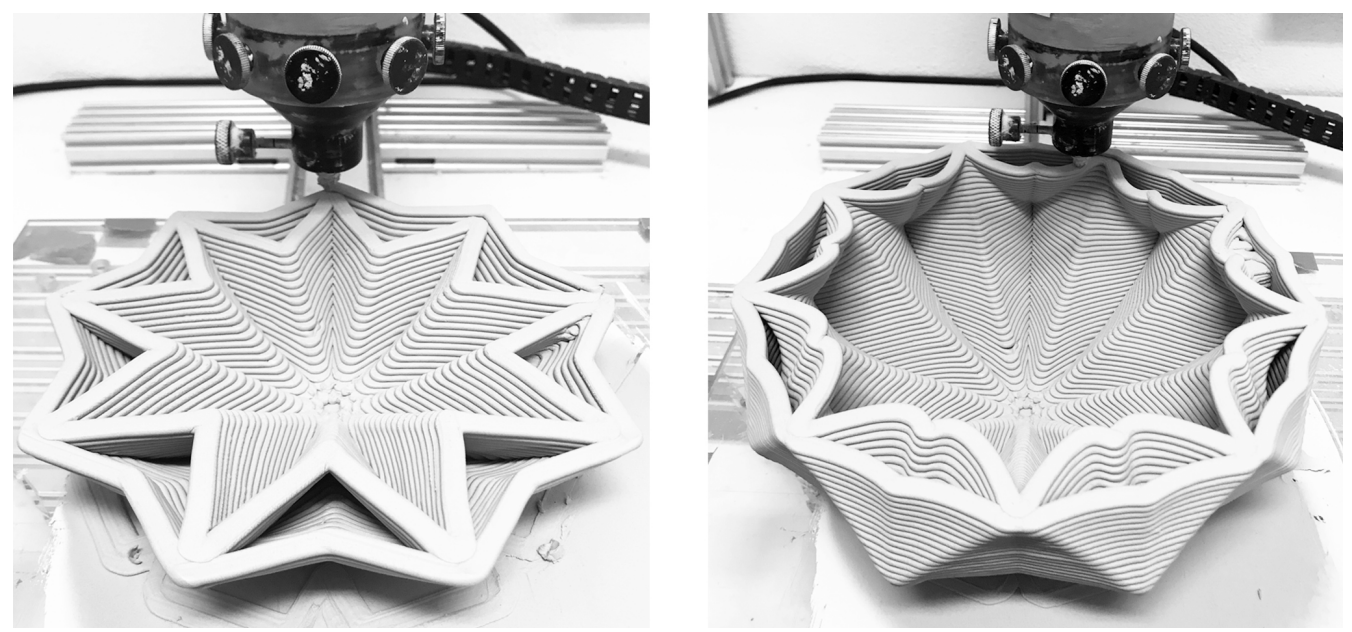

Figure 4

Detail views of a

print in process.

The concentric

surfaces intersect at critical moments and create lateral stability.

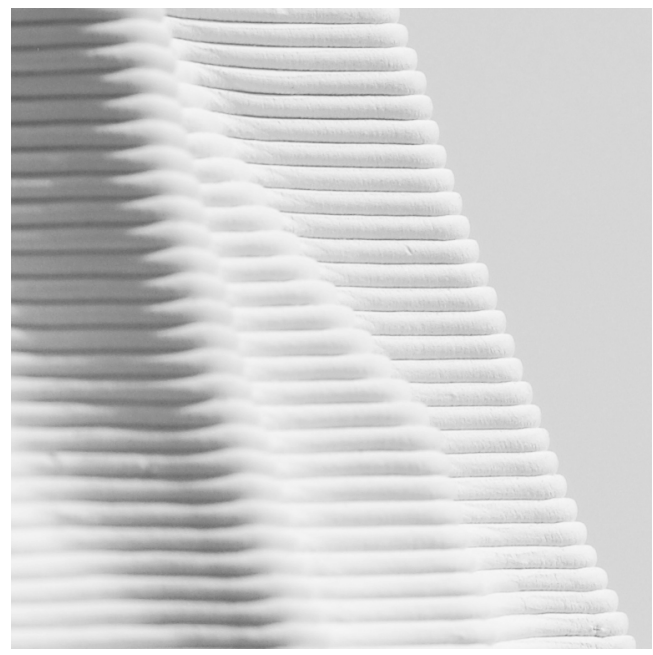

Figure 5

Staircasing affect from a $4 \mathrm{~mm}$ nozzle tip with a $2 \mathrm{~mm}$ z-step 
smooth surfaces before bisque firing or sintering occurs. This process is known as green machining and is heavily employed in the rapid prototyping of ceramic components (Riedel 2011). Green machining introduces its own array of unpredictable flaws like chipping, cracking and edge retention as the material is very fragile in this phase (Riedel 2011). The consistent recurring presence of these material flaws is one of the major hurdles researchers and manufactures are attempting to overcome. A majority of these flaws are not necessarily removed during the firing phases and are therefore present in the final product (Riedel 2011).

Minor surface defects that are not removed during the green machining phase can be further diminished through glazing techniques. Glazing produces a highly resilient waterproof layer that is generally desired in architectural applications. There is a material thickness that is added with ceramic glaze which needs to be accounted for in joint tolerances. This added material thickness coupled with the varying degrees of shrinkage during the firing stages are what make this material historically imprecise. The glazing material thickness is also what smoothens over minor surface defects. During this case study two glazing techniques were tested for consistency. Dip glazing and airbrushing were both tested on several iterations in varying degrees of thickness. It both instances the base of the artifact was coated with wax to prevent the glaze from adhering to the base. This ensures that the glaze will not fuse the object to the kiln shelves during the firing process. Alternatively, kiln spurs can be utilized if one requires the object to be entirely glazed. Ultimately, airbrushing the glaze provided a higher level of control over the dip technique. Applying three light coats with the airbrush produced a high-quality finish without adding substantial thickness.

Traditional kiln firing and sintering of aqueous ceramic paste prints proves to be challenging as substantial shrinkage occurs, which at times leads to cracking (Faes 2015). Calibrating this shrinkage has proven difficult in mass customization projects as each component is usually varied in its material composition and structure. This is why traditional modes of production such as extrusions and slip casting of uniform geometries continue to be the dominate techniques utilized in ceramic manufacturing. Highly engineered composite pastes that minimize shrinkage and manufacturing flaws continue to show the greatest potential for additive manufacturing of mass customized ceramic parts. However, many of these newly formulated pastes have yet to be implemented beyond the research phase (Feilden 2016). This research is critical if robocasting is to be used industrially where defect distributions are central to understanding a part's reliability (Feilden 2016).

The time required in the firing sequence also proves to be an ever-present constraint in the additive manufacturing of ceramics. Each artifact produced in this case study took a minimum of five days to produce. This process consisted of (in sequential order): the wet printing phase, 24-hour dry time, green machining, 12-hour bisque fire, 8-hour cooling, glaze application, 24-hour glaze fire and subsequent cooling. The factor of time in the manufacturing of ceramics is being tested through various other alternative modes of additive manufacturing. Stereolithography (SLA) has shown promise with employing a UV-curable resin as a binder which is mixed with the ceramic powder to create an engineered paste (Faes 2015). In this process the resin is cured by means of a UV-laser as it is dispersed. This instantaneously generates a green product, cutting out the bisque firing and cooling phases. The printed green body is then machined, glazed (if desired) and fired. While this dramatically reduces the overall time required, it is both cost- and material-inefficient (Faes 2015).

\section{CONCLUSION}

In conclusion, the imprecision of robocasting ceramics has limited the employment of ceramics in mass customized parts and components across the architectural profession. The robust material properties and cost effectiveness of ceramics are undeniable. 


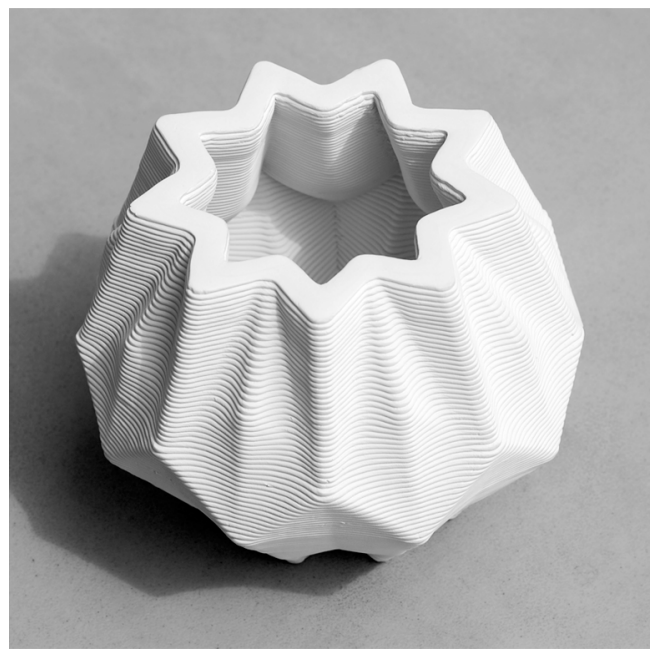

Figure 6

Subject artifact

after green

machining and

subsequent bisque

firing.

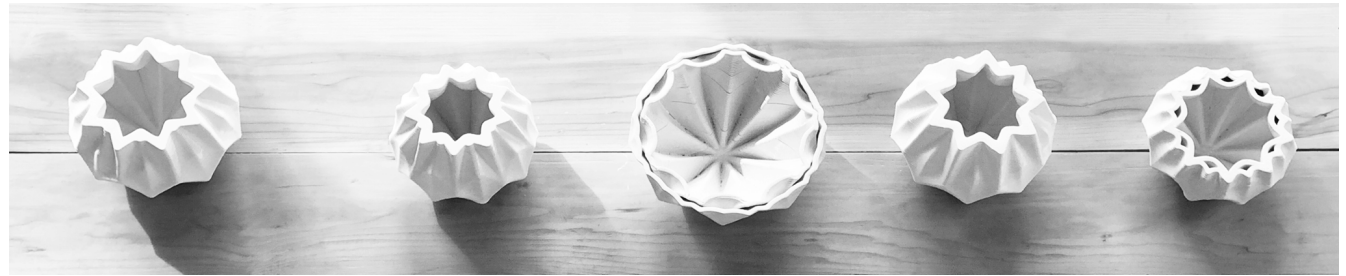

Figure 7

Case study

iterations

Rapid advances are being made in ceramic paste/slurry compositions as well as in the sintering processes that minimize material imprecision and time duration. However, these processes still prove to have technical issues and are cost prohibitive at the scale of architectural components. Advances are quickly being made, and therefore is only a matter of time before these hindrances are removed where additive manufacturing technologies become widely adopted across the ceramics industry and subsequently, the architecture community.

\section{REFERENCES}

Faes, M, Valkenaers, H, Vogeler, F, Vleugels, J and Ferraris, E 2015, 'Extrusion-based 3D Printing of Ce- ramic Components', Procedia CIRP, 28, pp. 76-81

Feilden, E 2017, Manufacturing of Ceramics and Ceramic Composites via Robocasting, Ph.D. Thesis, Imperial College London - Center for Advanced Structural Ceramics

Feilden, E, Blanca, EGT, Giuliani, F, Saiz, E and Vandeperre, L 2016, 'Robocasting of structural ceramic parts with hydrogel inks', Journal of the European Ceramic Society, 36, pp. 2525-2533

Riedel, R and Chen, IW (eds) 2011, Ceramics Science and Technology, Volume 3: Synthesis and Processing, Wiley-VCH

Smay, JE, Cesarano, J and Luis, JA 2002, 'Colloidal Inks for Directed Assembly of 3-D Periodic Structures', Langmuir, 84, pp. 5429-5437 